\title{
MÉTHODE DE DÉTERMINATION DE L'INDEX GÉNOTYPIQUE DES TAUREAUX D'INSÉMINATION SUR LA VALEUR DE LEURS VEAUX DE BOUCHERIE
}

\author{
B. VISSAC \\ avec la collaboration technique de G. Steier, et J. C. Deotte (') \\ Station contrale de Génétique animale, \\ Centre national de Recherches zootechniques, Jouy-en-Josas (Seine-et-Oise)
}

SOMMAIRE

La mise à l'épreuve sur descendance des taureaux de races à viande, utilisés par les centres d'insémination en croisement industriel, pour la production de veaux de boucherie, s'est généralisée au cours des ro dernières années dans la partie sud de la France.

Le calcul des index génotypiques des taureaux d'après la valeur de leurs veaux est effectué en 2 étapes :

- détermination des effets moyens de chaque taureau sur les critères envisagés. (Ces valeurs sont les estimées de moindres carrés obtenues en tenant compte des influences du sexe des reaux, de la race et du format des mères) ;

- estimation du génotype des taureaux après pondération des effets moyens ci-dessus par le coefficient de régression du génotype du taureau sur l'effet moyen correspondant.

Tous les calculs sont réalisés entièrement par voie mécanographique; on tient compte en particulier, dans cette réalisation, des variations dı schéma d'observation qui peuvent se présenter en pratique d'un centre à un autre.

\section{INTRODUCTION}

Dans une étude précédente (VISSAC et coll., I959) nous avons décrit les méthodes pratiques de mise à l'épreuve des taureaux d'insémination sur la valeur de leurs veaux de boucherie; nous avons interprété quelques résultats obtenus par les premiers centres français ayant appliqué ces méthodes (Puy-de-Dôme et Tarn-Aveyron) et proposé une formule simple de calcul de l'index génotypique de chaque taureau valable dans le cas d'un schéma expérimental balancé.

(1) Qui ont assuré la réalisation des programmes de calcul. 
Depuis, le testage des taureaux s'est développé dans la plupart des centres français situés dans des zones de production de veaux de boucherie suivant une méthode de contrôle simplifiée. L'importance des données recueillies dans ce sens et les possibilités nouvelles offertes par l'atelier mécanographique de la Station de Génétique animale nous ont conduit à définir une méthode rigoureuse de calcul de l'index génotypique des taureaux, applicable à un schéma non balancé et à assurer la programmation de ces calculs.

I a présente note a pour but de préciser cette méthode et d'en étudier les possibilités d'application suivant les situations particulières qui se présentent en pratique dans les centres d'insémination artificielle.

\title{
PRINCIPE GÉNÉRAL DE LA MÉTHODE
}

\author{
I. - NATURE DE' L'INFORMATION RECUEILLIE \\ ETT CARACTÉRISTIQUES DU SCHÉMA EXPÉRIMENTAL,
}

Les taurea11x mis à l'épreuve (đésignés par $T_{i}$ ) effectuent des inséminations d'essai pendant une période de temps limitée et dans une zone où les veaux sont en général commercialisés entre 6 semaines et 4-5 mois. Les naissances et les ventes des veaux issus des divers taureaux sont donc concentrées dans le temps et dans l'espace ce qui, joint au régime alimentaire intensif qu'ils subissent, élimine partiellement les fluctuations aléatoires des critères considérés, fluctuations liées au milieu de production ou aux conditions économiques. Le nombre de veaux contrôlés par taureau est de l'ordre de 50 ; ce nombre est celui qui correspond à la rentabilité maximum de 1'opération financière entreprise par le centre qui pratique le testage (Poutous et VISSAC, rg62).

Le cheptel femelle utilisé est souvent constitué de vaches de races variées désignées par $\mathrm{R}_{j}(j=\mathrm{I}, \ldots \mathrm{J})$. L’âge des femelles n'est en général pas connu. On caractérise leur format à l'aide d'une mensuration corporelle : le tour de poitrine $(\mathrm{Y})$.

Nous désignerons par $\mathrm{S}_{k} 1$ s sexe des veaux : $(k=I, 2)$.

I es contrôles effectués sur les veaux en vue de mesurer leur aptitude sont les suivants :

- contrôle de la croissance par pesées périodiques (mensuelles en général)

- contrôle de valeur bouchère réalisé subjectivement sur l'animal vif, par un expert, après notation des 7 points suivants :

Musculature de l'épaule,

Musculature du dos et du rein,

Musculature de la cuisse,

Finesse de l'os,

État d'engraissement,

Importance réduite du ventre,

Couleur des muqueuses (vulve et oil)

- contrôle de la valeur commerciale à partir du prix de vente unitaire et du cours moyen du marché (les veaux sont vendus au $\mathrm{kg}$ vif sur d'importants marchés locaux). 
Les données fournies par ces contrôles permettent de caractériser l'aptitude du veau à partir des 3 critères suivants :

${ }^{1} \mathrm{X}$ - Somme des 7 notes de valeur bouchère donnéas par l'expart à un âge voisin de l'âge commercial : les appréciations sont transformées, chacune, en une note comprise entre o et Io. (critère subjectif de valeur bouchère).

${ }^{2} \mathrm{X}$ - Prix de vente unitaire - cours moyen du marché correspondant (critère commercial de valeur bouchère).

${ }^{3} \mathrm{X}$ - Poids à un âge standard voisin de l'âge commercial moyen des veaux obtenu par intrapolation linéair ? entre les 2 pasé ss encadrant cat âge ou extrapolation au-delà de la dernière pesé s sur une périoda maximum de ro jours. (Critère caractérisant la vitesse de croissance).

\section{II. -- ESTMMATION DU PHINOTYYPL MOYEN DES VEAUX IH CHAQUE TAURLAU}

D'après le schéma expérimental exposé ci-dssus, las critères étudiés $\left({ }^{1} \mathrm{X},{ }^{2} \mathrm{X}\right.$, ${ }^{3} \mathrm{X}$ ), pour un reau donné, peuvent être considérés comme des variables aléatoires soumises à l'action de plusisurs factsurs de variation suivant le modèle suivant:

$$
\mathrm{X}_{i j k l}=u+t_{i}+r_{j}+s_{i j}+\varepsilon_{i j k i}
$$

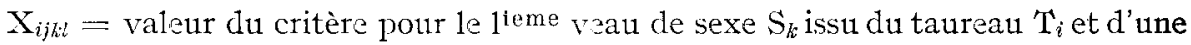
vache de race $R_{j}$.

$\mu=$ constante représentant la valeur moyenne du critère pour l'ensemble des veanx.

$t_{i}=$ effet moyen du taurau ' $\mathrm{T}$.

$r_{j}=$ effet moyen de la raca $\mathrm{R}_{j}$.

$s_{k}=$ effet moyen particulier au sex $s_{k}$.

$\varepsilon_{1 h l}=$ variable aléatoire $\mathrm{d}$ ? moyennz nulle ef $\mathrm{d}$ z variance $\sigma_{\varepsilon}^{2}$.

Le phénotype moyen des veaux du taureau ' $\mathfrak{L}_{i}$ pzut être caractérisé par $t_{i}$ ou $\hat{\mu}+t$ : suivant que l'on considère les critères en valıur brute ou les déviations par rapport à la moyenne estimé

Nous supposons, en adoptant ca modèle, q11 1 1 s effets des facteurs sont additifs, ce qui n'est vrai qu'en première approximation, 1əs variations du poids, notammant, semblant plutôt soumises à des influences multiplicatives.

Ce modèle ne tient pas compte, par ailleurs, des interactions entre les facteurs consiclérés et notamment pour ca qui est $d \geq 1$ 'influanca du taureau $T$ :

de l'interaction entre taureau et sex?,

de l'interaction entre taureau et race $\mathrm{d}=$ mère.

Nous ne nous préoccupons done que de l'effet moyen du taureau en croisement avec les diverses races de femelles (aptitude générale au croisement), éliminant de ce fait les effets particuliers liés aux différences de valeur entre les divers types de veaux croisés issus d'un même père (aptitude spécifique au croisement). Cette interaction ne pourra ètre établie pour chaqua taureau que sur un effectif de veaux par géniteur, plus important. 
Les estimées des moindres carrés des paramètres du modèle ci-dessus $\left(\hat{\mu}, \hat{\mathrm{t}}_{i}, \hat{\mathrm{r}}_{j} \hat{\mathrm{s}}_{k}\right)$ sont les solutions rendant minimum $\sigma_{\varepsilon}^{2}$ ou telles que :

$$
\underset{i j h l}{\Sigma}\left(\mathrm{X}_{i j k l}-\mu-t_{i}-r_{j}-s_{i}\right)^{2} \quad \operatorname{minimum}(\mathrm{I})
$$

Ces solutions sont celles du système d'équations obtenu en annulant les dérivées de l'expression (I) successivement par rapport à chacun des paramètres $\mu, t_{i}, r_{j}, s_{k}$ (équations normales) Ce système d'équations est représenté ci-dessous sous forme matricielle pour un critère $\mathrm{X}$.
$\hat{\mu}$
$\hat{t_{i}}$
$\hat{r}_{j}$
$\hat{s_{k}}$

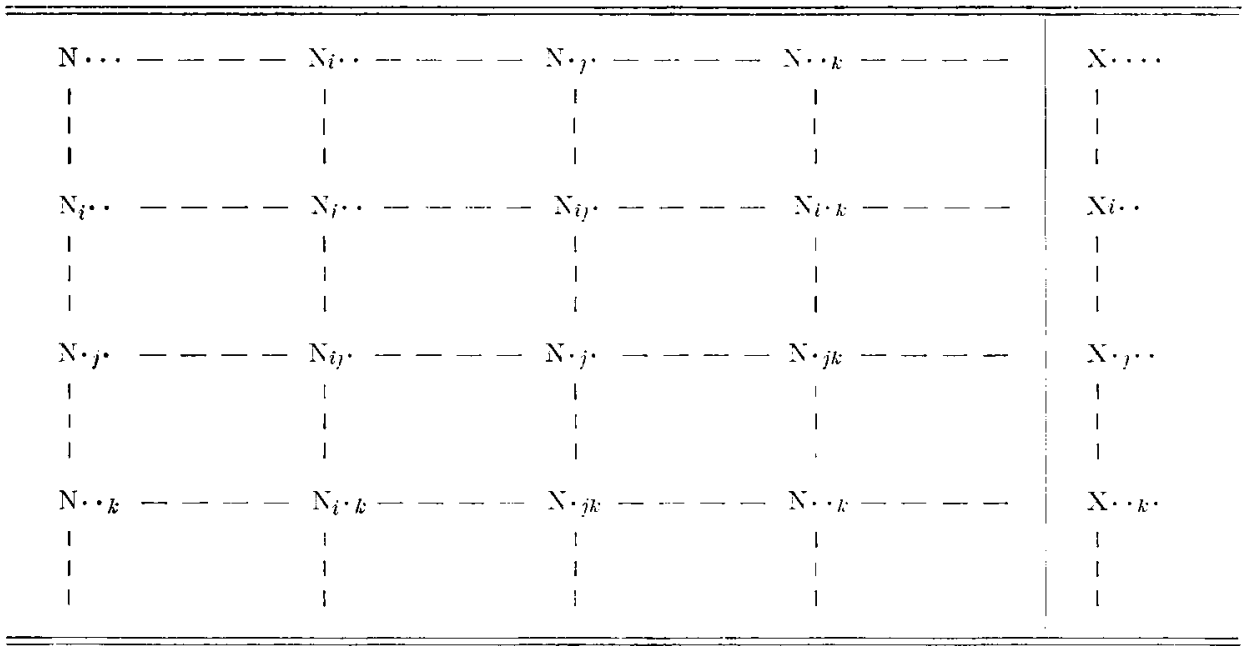

N... est l'effectif total des individus contrôlés pour le caractère $\mathrm{X}$.

$\mathrm{N}_{\boldsymbol{i} . .,}, \mathrm{N}_{\text {. } .,} \mathrm{N}_{\text {.. } \boldsymbol{k}}$ sont les effectifs par taureau $\mathrm{T}_{i}$, par race de mère $\mathrm{R}_{i}$ et par sexe $\mathrm{S}_{k}$ respectivement.

$\mathrm{N}_{i j} . \mathrm{l}^{\prime}$ effectif des veaux issus du taureau $\mathrm{I}^{\prime}$ i et des femelles de race $\mathrm{R}_{j}$ (idem pour N. ${ }_{j k}$ et $\left.\mathrm{N}_{i \cdot k}\right)$.

$\mathrm{X}$... est la somme totale des $\mathrm{X}$.

$\mathrm{X}_{i} \ldots, \mathrm{X}_{j}, \ldots, \mathrm{X} . \mathrm{X}_{k}$. les sommes relatives aux veaux du taureau $\mathrm{I}_{i}$, issus de raches de race $R_{j}$ et de sexe $S_{k}$ respectivement

Chaque ligne du tableau ci-dessus représente les coefficients des inconnues: $\hat{\mu}, \hat{t}_{i}, \hat{r}_{j}, \hat{s}_{k}$, dans une équation du système et la valeur du second membre de l'équation. Ainsi, pour la $\mathrm{I}^{\text {ere }}$ ligne:

$\mathrm{N} \cdots \mu+----+\mathrm{N}_{i} \cdot \hat{t}_{i}+---+\mathrm{N} \cdot{ }_{j} \cdot \hat{r}_{j}---+\mathrm{N} \cdots \hat{k}_{k}---=\mathrm{X} \cdots$

La résolution du système implique de fixer plusieurs conditions complémentaires ; on remarque en effet que ces équations ne sont pas indépendantes entre elles. Si I, 
$\mathrm{J}, \mathrm{K}$ représentent le nombre de taureaux, de taces de mère et de sexes, on dispose de $\mathrm{I}+\mathrm{J}+\mathrm{K}+\mathrm{I}$ équations dont

la somme des $\mathrm{K}$ dernières,

la somme des $\mathrm{J}$ précédentes,

la somme des I précédentes,

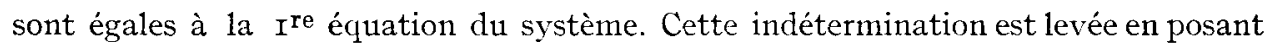
3 conditions complémentaires :

$$
\begin{aligned}
& \text { soit } \Sigma \hat{t}_{i}=\Sigma r_{j}=\Sigma \hat{s}_{k}=0 \\
& \text { soit } \hat{t}_{I}=\hat{r}_{J}=s_{K}=0 .
\end{aligned}
$$

Cela exprime que les effets moyens relatifs aux divers niveaux d'un facteur $\left(\hat{t}_{i}\right.$ par exemple) ne sont définis qu'à une constante près : on peut les exprimer en déviations par rapport à l'effet moyen d'un niveau donné $\left(\hat{t}_{\mathrm{I}}=0\right)$ ou par rapport à la moyenne des effets de l'ensemble des niveaux du facteur considéré $\left(\Sigma \hat{t_{i}}=0\right)$; I a r ${ }^{\text {ere }}$ solution a été retenue pour des raisons de simplicité des calculs.

La résolution du système de $\mathrm{I}+\mathrm{J}+\mathrm{K}+\mathrm{I}-3$ équations résultantes peut être réalisée par diverses méthodes dontla plus générale, sinon la plus rapide, est obtenue par voie matricielle :

Si $N$ représente la matrice carrée des effectifs, après simplification par les conditions ci-dessus, et $\mathrm{X}$ le vecteur des sommes $\mathrm{X} \ldots \mathrm{X}_{i} \ldots \longrightarrow$ (etc) le système peut s'écrire :

$$
\mathrm{N}]\left\|\begin{array}{c}
\hat{\mu} \\
\hat{t}_{i} \\
\hat{r}_{j} \\
\hat{s}_{k}
\end{array}\right\|=\| \mathrm{X}
$$

le vecteur solution :

$$
\left\|\begin{array}{c}
\hat{\mu} \\
\hat{t_{i}} \\
\hat{r}_{j} \\
\hat{s}_{h}
\end{array}\right\|=[\mathrm{N}]^{-1}\|\mathrm{X}\|
$$

est obtenu après inversion de la matrice des effectifs $[N]$ et multiplication de $[N]^{-1}$ par le vecteur des sommes $\|\mathrm{X}\|$

Les valeurs de $\hat{t}_{i}$ ou $\hat{\mu}+\hat{t}_{i}$ tirées du vecteur solution caractérisent le phénotype moyen des veaux du taureau $\mathfrak{T}_{i}$. Ces estimées étant définies par rapport â $\hat{t}_{\mathbf{r}}=$ o nous les avons recentrées pour la suite de l'étude, de façon que $\Sigma \hat{\bar{t}}_{i}=0$. Nous avons, par ailleurs, rapporté ces déviations à la moyenne générale de la variable dans l'échantillon $(\overline{\mathbf{X}} . .$.

N. B. - Le format des mères mesuré par leur tour de poitrine est également considéré comme une cause (contrôlée) de variation de ${ }^{3} \mathrm{X}$ - Dans un but de simplification des calculs nous avons corrigé cette influence en deux étapes :

$\left.\mathrm{I}^{\mathrm{e}}\right)$ Les estimées des moindres carrés des paramètres du modèle ci-dessus ont été également calculées pour le tour de poitrine des mères.

$\left.2^{e}\right)$ Les valeurs de $\hat{t}_{i}$ obtenues pour chaque taureau $\mathrm{T}_{i}$ en ce qui concerne ${ }^{3} \mathrm{X}$, ont été corrigées à partir de ces estimées en utilisant le coefficient de régression de ${ }^{3} \mathrm{X}$ sur $\mathrm{Y}$ obtenu intra-race de mère $(b)$. 


\section{III. - Estimation DE L'index GÉNOtYPiQUE DF, Chaque TAUREAU}

Si $\mathrm{I}_{i}$ représente l'index génotypique du taureau $\mathrm{T}_{i}$ :

$$
\mathrm{I}_{i}=\overline{\mathrm{X}} \cdots+b_{I t}\left(\hat{t}_{i}\right)
$$

$b_{1 t}$ étant le coefficient de régression du génotype sur le phénotype moyen, coefficient dont l'expression en fonction de l'effectif des descendants contrôlés $\left(n_{i}\right)$ et de l'héritabilité du caractère $\left(h^{2}\right)$, est connue :

$$
\begin{gathered}
b_{\mathbf{1}^{t}}=\frac{0,5 n_{i} h^{2}}{\mathrm{I}+\left(n_{i}-\mathrm{I}\right) 0,25 h^{2}} \\
\mathrm{I}_{i}=\overline{\mathrm{X}} \cdots+\frac{0,5 n_{i} h^{2}}{\mathrm{I}+\left(n_{i}-\mathrm{I}\right) 0,25 \bar{h}^{2}} \hat{t}_{i}
\end{gathered}
$$

\section{Application et réalisation des calculs}

En pratique, l'information recueillie par les centres effectuant le testage varie, le programme général de contrôle défini ci-dessus n'étant pas toujours appliqué de façon complète.

Dans la réalisation des calculs d'index génotypique par voie mécanographique, on a dû tenir compte de toutes les modalités de contrôle possibles.

Après les avoir passées en revue et indiqué les modifications de calcul qu'elles entraînent nous décrirons sommairement l'organigramme général des opérations mécanographiques. Les calculs sont effectués sur ordinateur IBM I620 à 20000 positions de mémoires

I) Modalités du contrôle.

Ces modalités varient pour une série de taureaux mis en testage à une époque donnée suivant :

- le nombre d'observations correspondant à chaque contrôle (N).

- les variables considérées $\left({ }^{1} \mathrm{X},{ }^{2} \mathrm{X},{ }^{3} \mathrm{X}, \mathrm{Y}\right)$

- les facteurs de classification $\left(\mathbf{T}_{i}, \mathrm{R}_{j}, \mathrm{~S}_{k}\right)$

A) Modalités concernant les variables. - ${ }^{1} \mathrm{X}$ : somme des 7 notes de pointage : en cas d'absence de l'une ou l'autre de ces notes :

- sur l'ensemble des veaux : on considère la somme des notes restantes ;

- sur quelques veaux seulement : on ne calcule pas ${ }^{1} \mathrm{X}$ pour ces veaux.

${ }^{2} \mathrm{X}$ : prix de vente - cours moyen du marché : en cas d'absence de ces 2 critères : on ne considère pas ${ }^{2} \mathrm{X}$. En cas d'absence du cours moyen mais non du prix de vente:

- sur l'ensemble des veaux; on considère le prix de vente comme valeur de ${ }^{2} \mathrm{X}$;

- sur quelques veaux seulement : ${ }^{2} \mathrm{X}$ n'est pas considéré pour ces veaux.

${ }^{3} \mathrm{X}$ : poids à un âge de référence. Cet âge doit être le plus près possible de l'âge d'abattage : âge variable suivant les veaux. En pratique, on calcule pour chaque veau le poids à des âges de références définis de $I_{5}$ j en $I_{5} j$. En consultant l'effectif des veaux à chaque âge de référence on choisit l'âge le plus élevé où subsistent au moins 
90 p. Ioo des veaux soumis au contrôle de croissance. Un âge plus élevé se rapprocherait certes mieux de l'âge moyen de commercialisation mais l'élimination d'un pourcentage élevé de veaux, pourcentage variable suivant les taureaux, risquerait d'entraîner une sélection importante des échantillons de veaux de chaque taureau.

B) Modalités concernant le nombre d'observations. - Pour toutes les raisons que nous venons de signaler : absence totale ou partielle d'un contrôle, abattage précoce d'un veau dont on ne peut calculer le poids à l'âge de référence choisi, le nombre d'observations varie suivant chaque critère. 'Tous les calculs définis ci-dessus: estimation statistique du phénotype moyen des veaux et de l'index génotypique doivent être réalisés séparément pour chaque variable ${ }^{1} \mathrm{X},{ }^{2} \mathrm{X},{ }^{3} \mathrm{X}$ et pour $\mathrm{Y}$.

C) Modalités concernant les facteurs de classification. $-\mathrm{R}_{j}$ : Les races des mères sont définies par la connaissance des 2 races parentales ce qui, étant donné le nombre de races et de types de croisement pratiqués dans certaines régions conduirait à considérer un nombre de niveaux $j$ parfois assez élevé et à effectuer des distinctions sans grand intérêt. Pour ériter cet écueil, on ne retient que les races ou types de croisement les plus représentés, les autres étant regroupés dans un niveau résiduel.

$\mathrm{S}_{k}$ : Étant donnée l'incidence de la gemellarité, sur la croissance notamment, il est apparu préférable d'isoler les jumeaux (mâles et femelles), quand ils existent, dans une classe spéciale, et d'inclure, pour les calculs, cet effet dans celui du sexe.

2) Réalisation des calculs.

Ies opérations mécanographiques effectuées sont les suivantes :

- Constitution du fichier de cartes perforées : ce fichier comporte pour chaque

TABLEAU 2

Calculs d'index génntypiques des taureanx du centre de...

\begin{tabular}{|c|c|c|c|c|c|c|c|c|}
\hline \multirow[b]{2}{*}{$\begin{array}{l}\text { Xuméro } \\
\text { du taureau }\left(\mathrm{T}_{i}\right)\end{array}$} & \multicolumn{3}{|c|}{$\begin{array}{l}\text { Effectifs des veaux } \\
\qquad \frac{\left(n_{i}\right)}{\text { Variable considérée }}\end{array}$} & \multicolumn{5}{|c|}{ Inflex génotypiques $\left(\mathrm{I}_{i}\right)$} \\
\hline & $3 \mathrm{X}$ & ${ }^{1} \mathrm{X}$ & ${ }^{2} \mathrm{X}$ & ${ }^{3} I_{i}$ & ${ }^{1} \mathrm{I}_{i}$ & ${ }^{2} \mathrm{I}_{i}$ & ${ }^{4} I_{i}$ & ${ }^{5} I_{i}$ \\
\hline $315550015700: 3$ & 033 & 029 & $0: 30$ & 09099 & 03998 & 55916 & 003997 & $05590 \%$ \\
\hline 3455500157005 & 071 & $06 i$ & 071 & 11069 & 03496 & $510: 39$ & $00_{1}^{\prime}+23$ & 059815 \\
\hline 3155500158002 & $0_{1}^{\prime}+5$ & $0: 1$ & $01: 3$ & 10079 & $0: 36118$ & 51067 & 1103646 & 051470 \\
\hline 8707700658001 & $0 \div 2$ & 037 & $0 \div 1$ & $113: 31$ & $03904 \%$ & 52178 & 00,123 & 059462 \\
\hline 'Total $(\mathrm{N} \cdots)$ & $199^{\prime}$ & 168 & 185 & & & & & \\
\hline Moyeme $(\bar{X} \ldots)$ & & & & 106010 & $0: 3880$ & 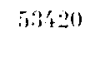 & 001112 & 056665 \\
\hline
\end{tabular}

Les résultats sont exprimés dans les unités suivantes :

$$
\begin{aligned}
& { }^{3} I_{i} \text { en décagrammes } \\
& { }^{1} I_{i} \text { en centiemes de note } \\
& { }^{2} I_{i} \text { en anciens centimes } \\
& { }^{4} I_{i} \text { en } \mathrm{kg} \times \text { note } \\
& { }^{5} I_{i} \text { en anciens francs }
\end{aligned}
$$


animal contrôlé, les éléments nécessaires à la connaissance des variables $\left({ }^{1} \mathrm{X},{ }^{2} \mathrm{X},{ }^{3} \mathrm{X}\right.$, ) et des facteurs de classification $\left(\mathrm{Y}, \mathrm{T}_{i}, \mathrm{R}_{j}, \mathrm{~S}_{k}\right)$

- Calcul des poids à des âges de référence par intrapolation linéaire entre les pesées et détection des erreurs (programme de calcul no 63007) ${ }^{1}$ ).

- Établissement du schéma d'analyse définitif. (variables et niveaux de chaque facteur) tenant compte des diverses modalités du contrôle, après un dépouillement sommaire des données de base (programme de calcul no 630r3)

- Constitution et résolution des systèmes linéaires (programmes no 63022 et 62006 )

- Correction de l'influence du format et calcul des index génotypiques pour chaque taureau $\mathrm{T}_{i}:{ }^{1} \mathrm{I}_{i},{ }^{2} \mathrm{I}_{i},{ }^{3} \mathrm{I}_{i}$ (programme 63024)

- Ce programme fournit également deux index économiques établis de façon empirique :

$$
\begin{aligned}
& { }^{4} I_{i}={ }^{1} I_{i} \times{ }^{3} I_{i} \\
& { }^{5} I_{i}={ }^{2} I_{i} \times{ }^{3} I_{i} .
\end{aligned}
$$

Les valeurs des index de chaque taureau d'une série de testage sont rassemblées sur un tableau ci-joint comportant également les effectifs de veaux contrôlés par taureau et par critère ainsi que les moyennes et effectifs de l'échantillon global.

\title{
CONCLUSION
}

Cette méthode de classement des taureaux d'insémination d'après les performances de leurs veaux doit être améliorée à l'avenir dans différentes directions.

I $^{0}$ - par la considération d'un modèle mathématique plus conforme tenant compte notamment de la valeur spécifique d'un taureau en croisement avec les. femelles de chaque race.

$2^{\circ}$ - par l'établissement d'index économiques plus objectifs dans lesquels on considèrera les corrélations génétiques entre les caractères étudiés.

\section{Reçu pour publication en avril 1964 .}

\section{SUMMARY}

\author{
METHOD OF DETERMINING THE GENOTYPIC INDEX OF A. I. BULLS \\ BY THE VALUE OF THEIR SLAUGHTER CALVES
}

The progeny-testing of beef-breed bulls, used by the A. I. centers in commercial cross breeding for veal production, was generalised in Southern France during the last ten years.

There are two steps in the calculations of the bulls' genotypic index by the value of their calves :

- determination of the average effects of each bull for the envisaged criteria. These values. are the overall estimates of least squares, allowing for the sex of the calves, the breed and dam's size ;

- estimation of the bulls' genotype, after weighing the above mentioned average effects, by the regression coefficient of the bulls' genotype on the average corresponding effect.

(1) Les numéros de programme indiqués sont des numéros de classement de la Station centrale de Génétique animale. 
All calculations are entirely undertaken on electronic computor. In the computing programs, we pay attention to any possible variations in the experimental plan which might occur in practice from one A. I. center to another.

\section{RÉFÉRENCES BIBLIOGRAPHIQUES}

Boyer J. P., 1958. Théorie et calcul des index de sélection. Ann. Zootech., 7, 193-242.

Poutous M., VIsSAC B., I962. Recherche théorique des conditions de rentabilité maximum de l'épreuve de descendance des taureaux d'insémination artificielle. Ann. Zootech., 11, 233-256.

Vissac B., Poly J., Charlet P., 1959. Les épreuves de descendance des taureaux d'insémination sur la valeur de leurs veaux de boucherie. Bull. tech. inf. Ing. Serv., Agric 145, 759-787. 